\title{
Performance of Early Outbreak Detection Algorithms in Public Health Surveillance from a Simulation Study
}

\author{
Gabriel Bedubourg*1,2 and Yann Le Strat ${ }^{3}$ \\ ${ }^{1}$ French Armed Forces Center for Epidemiology and Public Health, Marseille, France; ${ }^{2}$ UMR 912 SESSTIM, Aix Marseille University, \\ Marseille, France; ${ }^{3}$ French Institute for Public Health Surveillance, Saint Maurice, France
}

\section{Objective}

Evaluate the performance of 8 statistical methods for outbreak detection in health surveillance with historical data.

\section{Introduction}

Early detection of outbreaks is crucial in public health surveillance in order to enable rapid control measures. Statistical methods are widely used for outbreak detection (1) but no study has proposed to evaluate and compare thoroughly the performance of these methods.

\section{Methods}

We tested 23 outbreak detection algorithms from a thorough simulation study. Time series were generated using a negative binomial model under 42 scenarios depending on different trends, baseline frequencies of reports, seasonalities (annual or biannual) and dispersion (2). The simulated dataset included 231,000 624-week time series (TS): 4,200 TS without outbreak, 16,800 TS with 3 outbreaks in the past only, 42,000 TS with current outbreak only and 168,000 TS with 3 past and 1 current outbreak. A current outbreak means that it occurred in the last 50 weeks. Each simulated outbreak varied in duration and amplitude with tuning coefficients: $\mathrm{k}_{1}=0,2,5$ or 10 for past outbreaks and $\mathrm{k}_{2}=1$ to 10 for current outbreaks.

We focused on 8 detection methods, some methods being only variants: CDC algorithm, RKI3, Bayes3, CUSUM GLM Rossi, OutbreakP, and GLR Poisson, which are implemented in the R Surveillance package (3), and a periodic Poisson GLM based algorithm. For each algorithm, we used the same tuning parameters for all TS.

Each method was evaluated through its false positive rate (FPR) and its probability of detection (POD: at least one alarm during the outbreak period), for the different scenarios and outbreak sizes. The nominal FPRs were 0.005 for all the analyses.

\section{Results}

For each of the 42 scenarios, Figure 1 represents the mean of FPRs with $\mathrm{k}_{1}=5$ and Figure 2 represents the POD with $\mathrm{k}_{2}$ varying from 1 to 10. Only the Farrington, periodic Poisson GLM, and RKI3 algorithms presented a POD $>80 \%$ for the largest outbreaks $\left(\mathrm{k}_{2}>8\right)$ combined with a $\mathrm{FPR}<20 \%$, for each scenario. Bayes 3 method presented high POD with a FPR $>20 \%$ for some scenarios. CDC algorithm presented heterogeneous POD depending on the scenario with a FPR $<20 \%$. For the other 3 methods, FPR and POD greatly depend on the scenario.

\section{Conclusions}

We presented a systematic assessment of performance of 8 outbreak detection algorithms using a simulated dataset, large enough to include time series observed in the real surveillance systems. We also simulated a high diversity of outbreaks in terms of amplitude and duration. Since no single algorithm has presented sufficient performance for all scenarios, combinations of methods must be investigated to achieve predefined minimum performance. Other criteria of performance should be proposed in order to improve the choice of algorithms to be implemented in the surveillance systems.
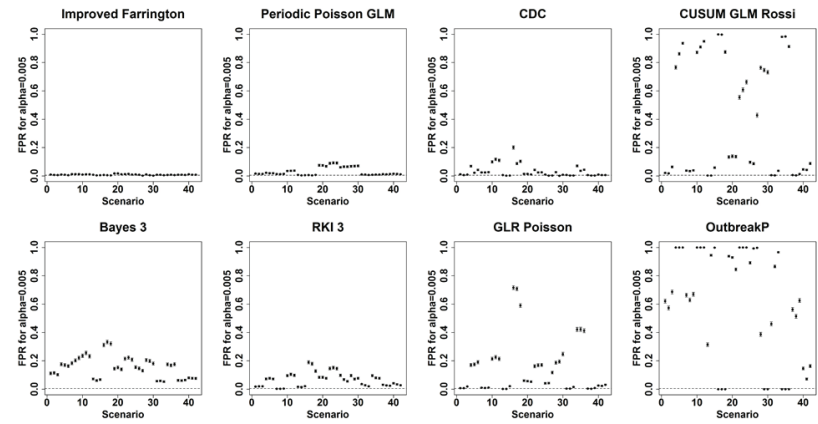

False positive rate for 8 outbreak detection algorithms (42 simulated scenarios - Past outbreak amplitude $\mathrm{k} 1=5$ )
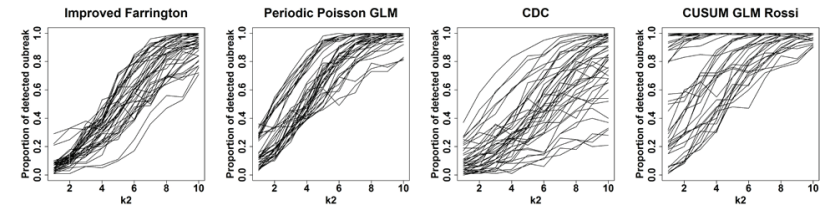

Bayes 3
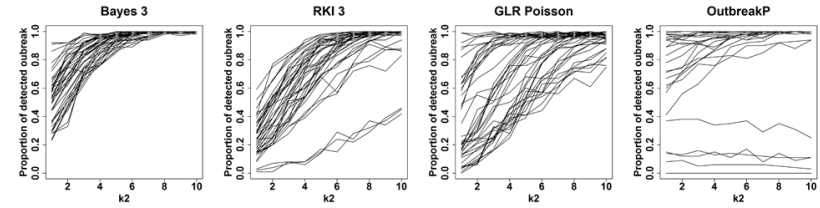

Probability of detection of 8 outbreak detection algorithms according to current outbreak amplitude (k2) (Past outbreak amplitude k1 = 5) (each curve corresponds to a simulated scenario)

\section{Keywords}

Health surveillance; Outbreak detection algorithm; Statistical method; Performance evaluation; Simulation study

\section{References}

1. Unkel S, Farrington CP, Garthwaite PH, Robertson C, Andrews N. Statistical methods for the prospective detection of infectious disease outbreaks: a review. J R Stat Soc Ser A Stat Soc. 2012;175(1):49-82.

2. Noufaily A, Enki DG, Farrington P, Garthwaite P, Andrews N, Charlett A. An improved algorithm for outbreak detection in multiple surveillance systems. Stat Med. 2013 Mar 30;32(7):1206-22.

3. Höhle M. Surveillance: An R package for the monitoring of infectious diseases. Comput Stat. 2007;22(4):571-82.

\section{${ }^{*}$ Gabriel Bedubourg}

E-mail: gabrielbedubourg@hotmail.fr 